\title{
Image-guided intensity-modulated radiotherapy improves short-term survival for abdominal lymph node metastases from hepatocellular carcinoma
}

\author{
Haige Zhang ${ }^{1, \#}$, Yixing Chen ${ }^{2 \#}$, Yong $\mathrm{Hu}^{2}$, Ping Yang ${ }^{2}$, Binliang Wang ${ }^{2}$, Jianying Zhang ${ }^{2}$, Jing Sun ${ }^{2}$, \\ Zhaochong Zeng \\ ${ }^{1}$ Department of Radiation Oncology, Luoyang Central Hospital Affiliated to Zhengzhou University, Luoyang 471000, China; ${ }^{2}$ Department of \\ Radiation Oncology, Zhongshan Hospital, Fudan University, Shanghai 200032, China \\ Contributions: (I) Conception and design: Z Zeng; (II) Administrative support: J Sun, Z Zeng; (III) Provision of study materials or patients: P Yang, \\ B Wang, J Sun; (IV) Collection and assembly of data: H Zhang; (V) Data analysis and interpretation: H Zhang, J Zhang, J Sun, Z Zeng; (VI) \\ Manuscript writing: All authors; (VII) Final approval of manuscript: All authors. \\ \#These authors contributed equally to this work. \\ Correspondence to: Jing Sun; Zhaochong Zeng. Department of Radiation Oncology, Zhongshan Hospital, Fudan University, 136 Yi Xue Yuan Road, \\ Shanghai 20032, China. Email: sun.jing@zs-hospital.sh.cn; zeng.zhaochong@zs-hospital.sh.cn.
}

Background: Radiotherapy (RT) is an effective treatment for hepatocellular carcinoma (HCC) patients with lymph node metastasis (LNM), which is a rare clinical situation with a poor prognosis. We evaluated the responses and toxicities in HCC patients with abdominal LNM treated with either image-guided intensitymodulated radiotherapy (IG-IMRT) or non-IG-IMRT.

Methods: Retrospective review of the records of HCC patients with regional LNM treated with IG-IMRT $(\mathrm{n}=43$ ) or non-IG-IMRT $(\mathrm{n}=42)$. The tumor responses, local control rates (LCRs), overall survival (OS) rates, and toxicities were evaluated.

Results: The mean biological effective dose with $\alpha / \beta=10$ Gy $\left(B_{10}\right)$ delivered to IG-IMRT group was $67.23 \pm 8.48$ vs. $63.43 \pm 5.01$ Gy delivered to non-IG-IMRT group $(\mathrm{P}=0.008)$. OS in IG-IMRT group vs. nonIG-IMRT group was 15.3 vs. 9.7 months $(\mathrm{P}=0.098)$. The one-year survival of IG-IMRT group was superior (69\% vs. 38.1\% for non-IG-IMRT, $\mathrm{P}=0.006$ ). Whereas two-year survival was not significantly different. Negative independent prognostic factors included $\geq 2$ positive lymph nodes and previous treatment without surgery, while $\mathrm{BED}_{10} \geq 65$ Gy was a protective factor. Toxicities were mild for both groups, while IG-IMRT group showed less late hepatotoxicity.

Conclusions: The therapeutic dose delivered by IG-IMRT is slightly higher than non-IG-IMRT which was more effective and showed superior short-term survival and local control in HCC patients with LNM.

Keywords: Lymph node metastasis (LNM); image-guided intensity-modulated radiotherapy (IG-IMRT); hepatocellular carcinoma (HCC)

Submitted Sep 03, 2019. Accepted for publication Nov 05, 2019.

doi: $10.21037 /$ apm.2019.11.17

View this article at: http://dx.doi.org/10.21037/apm.2019.11.17

\section{Introduction}

Lymph node metastasis (LNM) from hepatocellular carcinoma (HCC) is a rare clinical situation, with an incidence of $5.1-7.5 \%$ reported in several large surgical series $(1,2)$ and possibly as high as $26-37 \%$ on the basis of an autopsy series (3-6). HCC with LNM has been classified as an advanced stage, together with portal vein invasion and distant metastasis [according to the Barcelona Clinic of Liver Cancer (BCLC) guidelines], and these factors are related to a dismal prognosis. However, there 
is no consensus on the best choice for HCC patients with regional LNM. Surgical resection, including lymphadenectomy, can be performed in select patients, but few patients are suitable candidates for surgery at diagnosis (7). Sorafenib, recommended by BCLC guideline, in its milestone clinical trial, showed insignificant survival benefit in the subgroup of LNM (8). Nowadays, external beam radiation therapy (EBRT) as a palliative treatment strategy is recommended for stage III HCC in the 2011 Chinese Guidelines for diagnosis and treatment of HCC (9) and the 2009 Korean Liver Cancer Study Group practice guidelines (10), which based on a series of studies on radiotherapy (RT) for HCC. RT is an effective treatment with excellent RT response rates for HCC patients with LNM, which have been reported in many studies (11-13).

A retrospective study in 2005 was performed to evaluate the role of 2D RT for HCC patients with LNM for the previous 7 years at our institution (12). The median survival of EBRT group was 9.4 months, while it was 3.3 months for non-EBRT group. As RT technology has advanced, our institution has treated HCC patients with LNM using either non-image-guided intensity-modulated radiotherapy (non-IG-IMRT) or IG-IMRT techniques. Therefore, we evaluated RT responses, overall survival (OS) and toxicities in the groups treated by image-guided versus non-imageguided techniques to compare the safety and outcomes of these techniques.

\section{Methods}

\section{Patients and diagnosis}

We performed a retrospective review of records and outcomes of 85 HCC patients with LNM treated at Zhongshan Hospital from July 2011 to December 2016. All patients consecutively entered into our study. Patients were diagnosed as having HCC following either pathologic validation or clinical criterion (9).

Of these 85 HCC patients with LNM, six patients' metastatic lymph nodes were recognized to be synchronous with the diagnostic interval of HCC and LNM less than 1 month. The patterns of abdominal LNM from HCC were evaluated using the criteria of Zeng's report (12), and were generally classified into three groups as follows: hepatic portal, peripancreatic, and paraaortic lymph nodes. The clinical diagnosis of LNM from computed tomography (CT) or magnetic resonance imaging (MRI) was made based on the following criteria: (I) the shortest diameter of the lymph node exceeded $1 \mathrm{~cm}$ and (II) the lymph node was contrastenhanced in three phases. A positron emission tomography and computed tomography (PET-CT) scan was optional in these patients.

All patients underwent pretreatment baseline evaluation, such as physical examination, chest radiography, complete blood cell count (CBC), liver function testing, serum chemistry, serum alpha-fetoprotein (AFP) for patients who were HCC positive, and contrast-enhanced abdominal CT scan and/or MRI.

\section{Treatment}

Patients selected treatment by IG-IMRT or non-IG-IMRT according to doctors' recommendations and the patients' intentions and provided written informed consent. Patients in non-IG-IMRT group treated by either 3D conformal RT (CRT) $(n=30)$ or IMRT $(n=12)$. Of the 85 HCC patients, 74 patients with hepatitis $\mathrm{B}$ virus received antiviral therapy before and during radiotherapy. A 4D-CT simulator with contrast was performed to access respiratory liver motion with abdominal compression and to determine the internal target volume (ITV) (14). The radiation dose was limited by organ at risk (OARs), primarily the gastrointestinal (GI) tract. The target volume included involved lymph nodes, with or without intrahepatic lesions, and their abdominal lymph drainage regions. Gross target volume (GTV) was defined as enlarged lymph nodes and intrahepatic tumor. The ITV for intrahepatic lesions was determined by the summation of GTVs on inspiration and expiration. The clinical target volume (CTV) of lymph nodes was defined as ITV with a $3-5 \mathrm{~mm}$ margin (15) plus the surrounding lymph drainage regions. The planning target volume (PTV) was defined as CTV plus a margin of $5 \mathrm{~mm}$ in non-IGIMRT group, and plus $3 \mathrm{~mm}$ in the IG-IMRT group.

IG-IMRT was delivered by helical tomotherapy (Hi-ART), and non-IG-IMRT was delivered by linear accelerator (Siemens ONCOR). The treatment plans were produced by physicists based on the dose parameters required by oncologists. The total radiation doses were translated to biological effective doses $\left(\mathrm{BED}_{10}\right)$ according to a linear quadratic model with $\alpha / \beta=10 \mathrm{~Gy}$, which allowed comparability among the two groups. The median $\mathrm{BED}_{10}$ of PTV was $65 \mathrm{~Gy}$ in this study. The mean $\mathrm{BED}_{10}$ of IG-IMRT and non-IG-IMRT were $67.23 \pm 8.48$ vs. $63.43 \pm 5.01 \mathrm{~Gy}$, respectively. Each patient was treated with five fractions per week. The prescribed dose was to cover $95 \%$ of an isodose curve. Patients in the IG-IMRT group underwent 
megavoltage computed tomography (MVCT) before each treatment to correct the displacement of all the target volumes from the original positions on previous simulation CT. Electronic portal imaging device measurements were performed in the non-IG-IMRT group once per week.

\section{Follow up}

Patients underwent blood monitoring for parameters such as $\mathrm{CBC}$, liver function, serum chemistry values, and AFP for patients who were positive at diagnosis as well as physical examinations once a week during radiotherapy. The tumor responses to EBRT were evaluated 1.5-2 months later with follow-up CT or MRI after completion of RT, and then evaluated every 3 months. The OS was calculated from the first day of RT treatment to either the last visit (September $15,2018)$ or the date of death. The data from last follow up for living patients, death for patients who died, or the last data prior to loss of follow up were considered as end point data.

\section{Response and toxicity evaluation}

Tumor response evaluation was performed following Response Evaluation Criteria in Solid Tumors (RECIST), Version1.1 (16), and done by two investigators and reviewed by an independent radiologist. Complete response (CR) was defined as complete radiologic disappearance of intrahepatic tumors in-field of radiation. However, CR for all the metastatic lymph nodes was defined as total vanishment or less than $1 \mathrm{~cm}$ remaining for each, based on clinical or radiographic evidence. If the decrease in the sum of the longest cross-sectional area of the target lesion reached $\geq 30 \%$ reduction, it was defined as partial response (PR). When the target lesions increased more than $20 \%$ in the sum of diameters, it was defined as progressive disease (PD). Stable disease (SD) was defined as a response between PR and PD.

Toxicity evaluation was based on Radiation Therapy Oncology Group (RTOG) criteria (17) and the criteria of radiation-induced liver disease (RILD). Acute toxicity was evaluated weekly during RT and within 3 months after RT while late toxicity was accessed 3 months later and within 1 year after EBRT. Hepatotoxicity induced by RT was defined as classic RILD and non-classic RILD. Classic RILDs are characterized by anicteric hepatomegaly and ascites 1-3 months after RT. Non-classic RILD is characterized by 5 -fold of the alanine aminotransferase (ALT) elevation $(18,19)$.

\section{Statistical analysis}

Data were expressed as the mean \pm standard deviation or median. The Kaplan-Meier method and log-rank test were applied to analyze OS. The $\chi^{2}$-test and Mann-Whitney $\mathrm{U}$ test were performed to compare the two groups with respect to baseline characteristics, response and toxicity evaluations. The Cox's regression model was used for multivariate analysis of survival. For multivariate analysis, variables in univariate analysis with a $\mathrm{P}$ value $\leq 0.1$ were entered in a single step, applying backward stepwise regression (likelihood ratio). A $\mathrm{P}$ value $<0.05$ was considered statistically significant. All statistical analyses were performed using SPSS 24.0 software (IBM Corp, Armonk, USA) for windows.

\section{Results}

\section{Patient characteristics}

The baseline summary of HCC patients with LNM included in this study is shown in Table 1 . There were 77 men and 8 women, and the median age was 52 years (range, $31-76$ years). Six patients $(7.1 \%)$ were diagnosed with HCC and LNM synchronously. Of the 85 patients, $18(21.2 \%)$ had tumor thrombosis, and $62(72.9 \%)$ had intrahepatic tumor together with LNM at the time of RT. Of those 62 patients, 44 (71.0\%) were treated for both intrahepatic tumor and metastatic lymph nodes. There were 48 patients $(56.5 \%$ ) who presented with $\geq 2$ metastatic lymph nodes. Patients in the IG-IMRT group $(n=42)$ and the non-IG-IMRT group ( $n=43)$ had similar characteristics.

\section{Dosage comparison and tumor response}

The radiation dose delivered to PTV by IG-IMRT was significantly higher than that delivered by non-IGIMRT. The mean dose of IG-IMRT $v s$. non-IG-IMRT was $56.02 \pm 7.06(1.8-5.2 \mathrm{~Gy} /$ fraction $)$ vs. $52.86 \pm 4.17 \mathrm{~Gy}$ (1.8-2.4 Gy/fraction), respectively $(\mathrm{P}=0.009)$. The $1-$ and 2-year local control rates (LCRs) for the two groups were significantly different $(\mathrm{P}=0.019)$, indicating an obvious advantage for the IG-IMRT group (Figure 1).

Prior to radiotherapy, three patients in the non-IGIMRT group and one in the IG-IMRT group complained of upper abdominal pain, and one patient per group suffered from jaundice before radiotherapy. After the completion of radiotherapy, pain was relieved in all and jaundice was completely cleared.

Figure 2 shows a representative case. The target volumes 
Table 1 Baseline characteristics in 85 hepatocellular carcinoma patients with lymph node metastasis

\begin{tabular}{|c|c|c|c|}
\hline Variables & $\begin{array}{c}\text { IG-IMRT } \\
(n=43)\end{array}$ & $\begin{array}{c}\text { Non-IG-IMRT } \\
(n=42)\end{array}$ & $P$ values \\
\hline \multicolumn{4}{|l|}{ Age (years) } \\
\hline Median \pm SD & $51.7 \pm 9.9$ & $52.6 \pm 10.6$ & 0.689 \\
\hline Range & $35-76$ & $31-74$ & \\
\hline Gender, n (\%) & & & 0.684 \\
\hline Female & $3(7.0)$ & $5(11.9)$ & \\
\hline Male & $40(93.0)$ & $37(88.1)$ & \\
\hline HBsAg, n (\%) & & & 0.967 \\
\hline Negative & $5(11.6)$ & $6(14.3)$ & \\
\hline Positive & $38(88.4)$ & $36(85.7)$ & \\
\hline KPS, n (\%) & & & 0.084 \\
\hline 80 & $6(14.0)$ & $8(19.0)$ & \\
\hline 90 & $37(86.0)$ & $31(73.8)$ & \\
\hline 100 & $0(0.0)$ & $3(7.1)$ & \\
\hline \multicolumn{2}{|c|}{ Child-Pugh classification, n (\%) } & & 0.485 \\
\hline A & $41(95.3)$ & $42(100.0)$ & \\
\hline B & $2(4.7)$ & $0(0.0)$ & \\
\hline AFP status $(\mu \mathrm{g} / \mathrm{L}), \mathrm{n}(\%)$ & & & 0.363 \\
\hline$\leq 20$ & $20(46.5)$ & $12(28.6)$ & \\
\hline $20-400$ & $7(16.3)$ & $10(23.8)$ & \\
\hline$\geq 400$ & $13(30.2)$ & $15(35.7)$ & \\
\hline Unknown & $3(7.0)$ & $5(11.9)$ & \\
\hline Tumor thrombosis, n (\%) & & & 0.955 \\
\hline Negative & $34(79.1)$ & $33(78.6)$ & \\
\hline Positive & $9(20.9)$ & $9(21.4)$ & \\
\hline Intrahepatic tumor, n (\%) & & & 0.859 \\
\hline Presence & $31(72.1)$ & $31(73.8)$ & \\
\hline Absence & 12 (27.9) & $11(26.2)$ & \\
\hline Symptom, n (\%) & & & 0.563 \\
\hline Pain & $1(2.3)$ & $3(7.1)$ & \\
\hline Jaundice & $1(2.3)$ & $1(2.4)$ & \\
\hline No & $41(95.3)$ & $38(90.5)$ & \\
\hline Synchronous LNM, n (\%) & & & 0.650 \\
\hline Presence & $2(4.7)$ & $4(9.5)$ & \\
\hline Absence & $41(95.3)$ & $38(90.5)$ & \\
\hline Site of LN , n (\%) & & & 0.798 \\
\hline Portal & $5(11.6)$ & $7(16.7)$ & \\
\hline Peripancreatic & $21(48.8)$ & $19(45.2)$ & \\
\hline Paraaortic & $17(39.5)$ & $16(38.1)$ & \\
\hline
\end{tabular}

Table 1 (continued)
Table 1 (continued)

\begin{tabular}{lccc}
\hline Variables & $\begin{array}{c}\text { IG-IMRT } \\
(\mathrm{n}=43)(\%)\end{array}$ & $\begin{array}{c}\text { Non-IG-IMRT } \\
(\mathrm{n}=42)(\%)\end{array}$ & P values \\
\hline $\begin{array}{l}\text { LN number, n (\%) } \\
\quad \text { Solitary }\end{array}$ & $18(41.9)$ & $19(45.2)$ & 0.754 \\
$\quad$ Multiple & $25(58.1)$ & $23(54.8)$ & \\
LN size $(\mathrm{cm})^{\dagger}, \mathrm{n}(\%)$ & & & 0.154 \\
$<5$ & $23(53.5)$ & $16(38.1)$ & \\
$\geq 5$ & $20(46.5)$ & $26(61.9)$ &
\end{tabular}

Before treatment of intrahepatic tumor, $\mathrm{n}(\%)$

0.595

\begin{tabular}{lrrr} 
Surgery & $27(62.8)$ & $23(54.8)$ & \\
Non surgery & $16(37.2)$ & $19(45.2)$ & \\
Radiation fraction, $\mathrm{n}(\%)$ & & & \\
Mean & $21.63 \pm 4.33$ & $26.05 \pm 1.97$ & $<0.001$ \\
Dose (Gy) & & & \\
Mean \pm SD & $56.02 \pm 7.06$ & $52.86 \pm 4.17$ & 0.009 \\
BED & & & \\
Mean \pm GD & & & \\
$<65, \mathrm{n}(\%)$ & $67.23 \pm 8.48$ & $63.43 \pm 5.01$ & 0.008 \\
$\geq 65, \mathrm{n}(\%)$ & $19(44.2)$ & $32(76.2)$ & \\
\hline
\end{tabular}

${ }^{\dagger}$, sum of the longest diameters of multiple lymph nodes. IGIMRT, image-guided intensity-modulated radiotherapy; HBsAg, hepatitis B surface antigen; KPS, Karnofsky Performance Status; AFP, $\alpha$-fetoprotein; TACE, transcatheter arterial chemoembolization; LNM, lymph node metastasis; LN, lymph node.

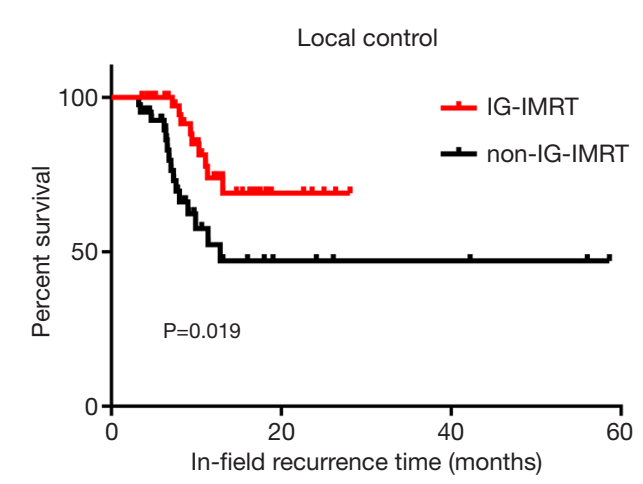

Figure 1 Local control of LNM in the IG-IMRT and non-IGIMRT group of HCC patients. IG-IMRT, image-guided intensitymodulated radiotherapy; LNM, lymph node metastasis; HCC, hepatocellular carcinoma. 

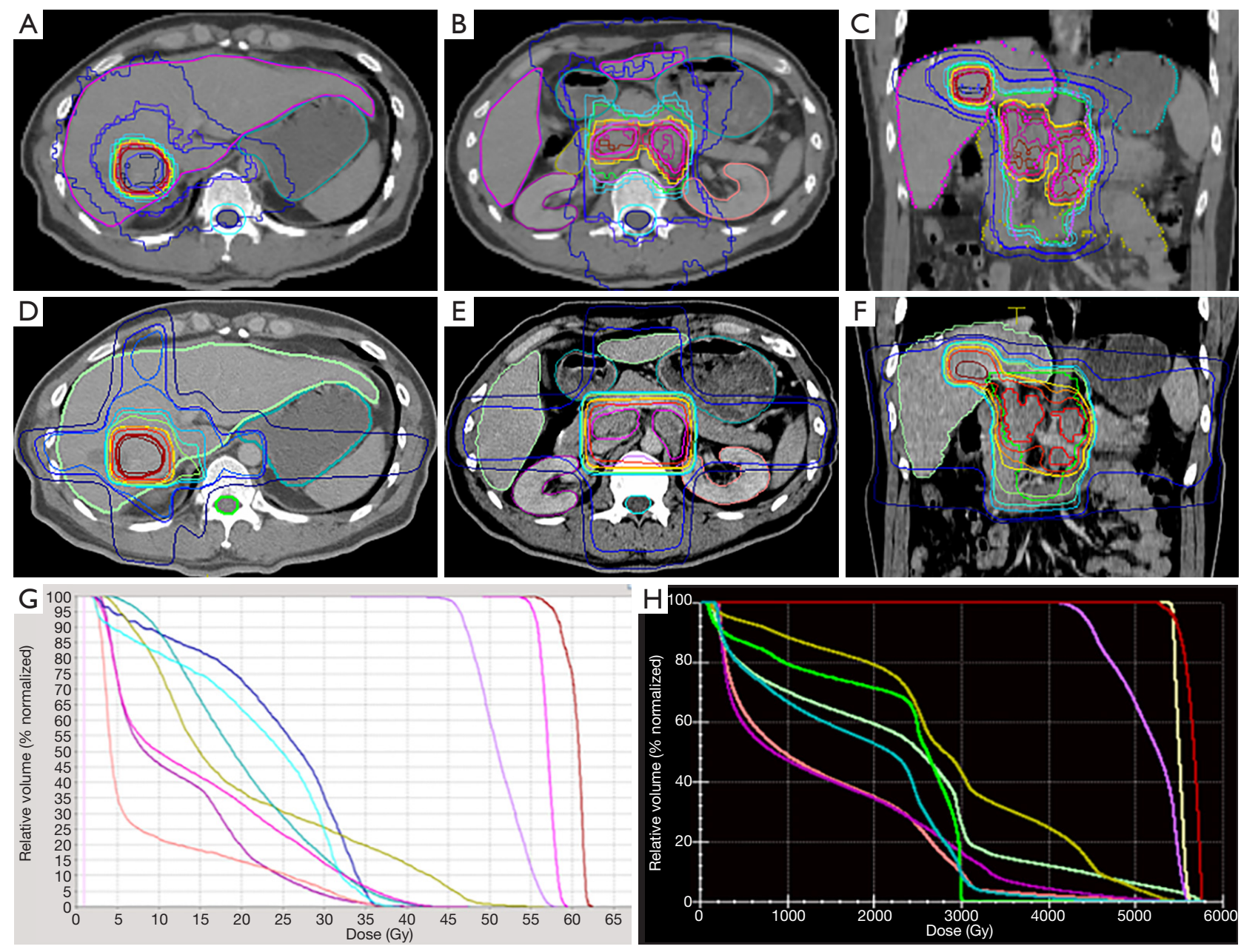

Figure 2 CT scan (arterial phase) for a 46-year-old man with right lobe HCC and paraaortic LNM. Two treatment plans based on the same target delineation were produced for IG-IMRT and 3D-CRT to compare dose distribution by physicist. (A,D) Dose distribution of intrahepatic tumor for IG-IMRT and 3D-CRT, respectively; (B,E) dose distribution of LNM for IG-IMRT and 3D-CRT, respectively; (C,F) dose distribution of all the lesions for IG-IMRT and 3D-CRT (shown in the coronal view); (G,H) DVH of IG-IMRT and 3D-CRT, respectively. CT, computed tomography; HCC, hepatocellular carcinoma; LNM, lymph node metastasis; IG-IMRT, image-guided intensitymodulated radiotherapy; 3D-CRT, conformal radiotherapy; DVH, dose-volume histogram.

of the GTV, PTV, and OARs were based on the same target delineation for both the IG-IMRT and 3D-CRT treatment plans. The dose data of the above target volumes, according to dose constrains are listed in Table 2, and indicate that IG-IMRT had an obvious advantage in protecting OARs, such as the normal liver (whole liver, excluding GTV), intestines, and kidneys (especially the left kidney for this patients). However, the maximal dose for the spinal cord for this patients' IG-IMRT plan was greater than that for the 3D-CRT plan with the permission of dose constrain of spinal cord. The conformal degree of IG-IMRT treatment was superior with PTV45, PTV55, and PTV57.5, compared to $3 \mathrm{D}-\mathrm{CRT}$.

\section{Survival analyses and prognostic factors}

At the end point, three patients in the IG-IMRT group $(n=43)$ and four patients in the non-IG-IMRT group $(n=42)$ were alive, and the others had died. The median OS of the IG-IMRT vs. non-IG-IMRT group was 15.3 vs. 9.7 months, respectively $(\mathrm{P}=0.098)$, the relative risk $(\mathrm{RR})$ value was 0.67 , and $95 \%$ CI was $0.42-1.08$. However, the 1 -year survival of the IG-IMRT group $v s$. non-IG-IMRT group was $69.1 \%$ vs. $38.1 \%$, respectively $(\mathrm{P}=0.006)$, which demonstrated a 
Table 2 Comparison of dose distribution of OARs for IG-IMRT and 3D-CRT treatment plan

\begin{tabular}{|c|c|c|c|}
\hline OAR & Dose type & IG-IMRT & 3D-CRT \\
\hline \multirow[t]{6}{*}{ Liver (normal) } & V30 (\%) & 23.8 & 25.6 \\
\hline & V20 (\%) & 41.2 & 58.7 \\
\hline & V10 (\%) & 55.8 & 69.7 \\
\hline & V5 (\%) & 75.7 & 78.2 \\
\hline & D max (Gy) & 62.4 & 57.7 \\
\hline & D mean (Gy) & 18.5 & 21.9 \\
\hline Spinal cord & D max (Gy) & 36.8 & 30.3 \\
\hline \multirow[t]{3}{*}{ Intestine } & V50 (\%) & 0.79 & 4.29 \\
\hline & D max (Gy) & 56.6 & 55.4 \\
\hline & D mean (Gy) & 20.3 & 28.7 \\
\hline \multirow[t]{3}{*}{ Stomach } & V50 (\%) & 0 & 0.02 \\
\hline & D max (Gy) & 48.4 & 52 \\
\hline & D mean (Gy) & 20.6 & 17.9 \\
\hline \multirow[t]{3}{*}{ Right kidney } & V22.5 (\%) & 16.9 & 30.7 \\
\hline & D max (Gy) & 56.3 & 54.8 \\
\hline & D mean (Gy) & 13.2 & 14.6 \\
\hline \multirow[t]{3}{*}{ Left kidney } & V15 (\%) & 22.9 & 41.1 \\
\hline & D max (Gy) & 51.02 & 53.71 \\
\hline & D mean (Gy) & 9.97 & 14.1 \\
\hline \multirow[t]{2}{*}{ PTV45 } & $\mathrm{Cl}$ & 0.65 & 0.20 \\
\hline & $\mathrm{HI}$ & 0.31 & 0.03 \\
\hline \multirow[t]{2}{*}{ PTV55 } & $\mathrm{Cl}$ & 0.46 & 0.18 \\
\hline & $\mathrm{HI}$ & 0.07 & 0.07 \\
\hline \multirow[t]{2}{*}{ PTV57.5 } & $\mathrm{Cl}$ & 0.52 & 0.18 \\
\hline & $\mathrm{HI}$ & 0.08 & 0.07 \\
\hline
\end{tabular}

OAR, organ at risk; IG-IMRT, image-guided intensity-modulated radiotherapy; 3D-CRT, conformal radiotherapy; $\mathrm{Cl}$, conformal index; HI, homogeneity index.

survival advantage of IG-IMRT irradiation. The survival of the IG-IMRT $v$ s. non-IG-IMRT group was $19.3 \%$ s. $14.5 \%$, respectively, at 2 years $(\mathrm{P}=0.066)$ (Figure 3$)$.

Univariate and multivariate survival analyses (Table 3) showed that $\geq 2$ lymph nodes (RR, 2.00, 95\% CI, 1.22-3.29, $\mathrm{P}=0.006)$ and previous treatment without surgery $(\mathrm{RR}, 1.71$, 95\% CI, 1.02-2.88, $\mathrm{P}=0.043$ ) are unfavorable prognostic factors associated with worse survival rates. The radiation

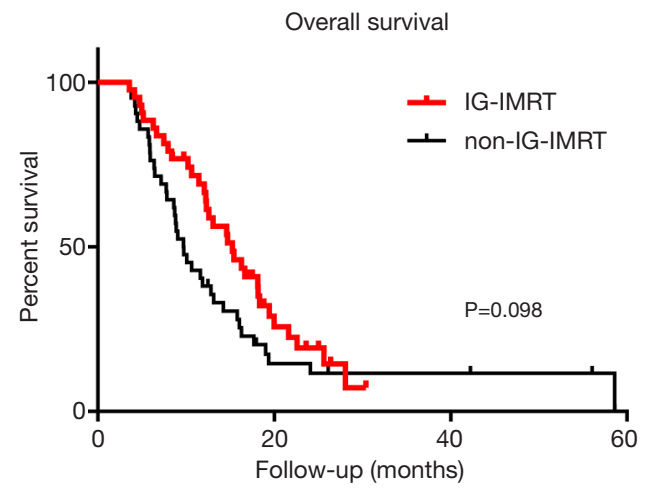

Figure 3 OS curve of the IG-IMRT and non-IG-IMRT group of HCC patients with LNM. IG-IMRT, image-guided intensitymodulated radiotherapy; OS, overall survival; HCC, hepatocellular carcinoma; LNM, lymph node metastasis.

dose $\mathrm{BED}_{10} \geq 65$ Gy was a protective factor $(\mathrm{P}<0.001$, RR, $0.32,95 \%$ CI, 0.19-0.54. A higher radiation dose, within the dose limits of OARs, indicated better survival rates. Table 3 provides detailed data of survival analyses and Cox regression analyses.

\section{Toxicity}

Treatment-related toxicities, such as acute and late GI toxicities, hematological toxicity, and acute hepatic toxicity, were observed at similar rates in both groups (Table 4). Acute toxicity and hematological toxicity were mild, with no acute toxicity greater than grade 4 toxicity observed in either group. Five total patients with elevated liver enzymes within 3 months after RT were considered to have acute liver reactions. Late hepatic toxicity was found to be similar between the two groups $(\mathrm{P}=0.366)$. Massive ascites (grade 3) were found within 6 months in 5 patients $(11.9 \%)$ in the non-IG-IMRT group $v s .2$ patients $(4.7 \%)$ in the IG-IMRT group. Three patients (7\%) in the IG-IMRT group and 1 patient $(2.4 \%)$ in the non-IGIMRT group were observed for hepatic encephalopathy (grade 4) and late hepatic toxicity, and suffered liver failure within 1 year after completion of radiotherapy. However, three patients died of grade 5 late hepatic toxicity in the non-IG-IMRT group, while only one patient did so in the IG-IMRT group.

\section{Cause of death}

Of the 78 patients who had died, 33 (42.3\%) died of liver 
Table 3 Univariate and multivariate analyses for overall survival

\begin{tabular}{|c|c|c|c|c|c|c|c|c|c|}
\hline Variables & $\begin{array}{c}\text { Patients } \\
(n=85)\end{array}$ & \multicolumn{4}{|c|}{ Kaplan-Meier survival } & \multicolumn{2}{|c|}{ Univariate analysis } & \multicolumn{2}{|c|}{ Multivariate analysis } \\
\hline Gender & & & & & 0.293 & & & & \\
\hline Female & 8 & 87.5 & 30.0 & 14.7 & & 1 & & & \\
\hline Male & 77 & 49.7 & 16.1 & 11.9 & & $0.62(0.25-1.53)$ & 0.298 & & \\
\hline$A$ & 83 & 53.4 & 17.3 & 12.3 & & 1 & & & \\
\hline B & 2 & 50.0 & 0 & 7.5 & & $1.94(0.47-8.02)$ & 0.360 & & \\
\hline Tumor thrombosis & & & & & 0.073 & & & & \\
\hline Presence & 62 & 52.5 & 45.3 & 12.2 & & 1 & & & \\
\hline Absence & 23 & 55.7 & 8.8 & 17.7 & & $2.45(1.31-4.60)$ & 0.005 & & \\
\hline Site of LN involvement & & & & & 0.124 & & 0.130 & & \\
\hline Portal & 12 & 41.7 & 25.0 & 9.7 & & 1 & & & \\
\hline Peripancreatic & 40 & 64.8 & 21.6 & 15.4 & & $0.77(0.38-1.53)$ & 0.451 & & \\
\hline Paraaortic & 33 & 43.4 & 8.3 & 11.6 & & $1.32(0.65-2.70)$ & 0.441 & & \\
\hline LN number & & & & & 0.087 & & & & \\
\hline Previous treatment & & & & & 0.001 & & & & \\
\hline Surgery & 46 & 61.0 & 22.8 & 14.7 & & 1 & & 1 & \\
\hline Non surgery & 39 & 36.7 & 3.3 & 10.1 & & $2.18(1.34-3.53)$ & 0.002 & $1.71(1.02-2.88)$ & 0.043 \\
\hline EBRT & & $P=0.006$ & $P=0.066$ & & 0.098 & & & & \\
\hline IG-IMRT & 42 & 69.1 & 19.3 & 15.3 & & 1 & & & \\
\hline Non-IG-IMRT & 43 & 38.1 & 14.5 & 9.7 & & $0.67(0.42-1.08)$ & 0.101 & & \\
\hline $\mathrm{BED}_{10}$ & & & & & $<0.001$ & & & & \\
\hline$<65$ & 51 & 37.3 & 5.7 & 9.7 & & 1 & & 1 & \\
\hline$\geq 65$ & 34 & 78.5 & 33.9 & 18.2 & & $0.34(0.20-0.58)$ & $<0.001$ & $0.32(0.19-0.54)$ & $<0.001$ \\
\hline
\end{tabular}

$\mathrm{RR}$, relative risk; EBRT, external beam radiotherapy. 
Table 4 Toxicity of radiotherapy delivered by IG-IMRT or nonIG-IMRT

\begin{tabular}{|c|c|c|c|}
\hline Side effect, RTOG grade & $\begin{array}{c}\text { IG-IMRT } \\
(n=43),(\%)\end{array}$ & $\begin{array}{c}\text { Non-IG-IMRT } \\
(n=42),(\%)\end{array}$ & $P$ value \\
\hline Acute gastrointestinal toxicity & & & 0.432 \\
\hline 0 & $7(16.3)$ & $4(9.5)$ & \\
\hline 1 & $28(65.1)$ & $26(61.9)$ & \\
\hline 2 & $8(18.6)$ & $12(28.6)$ & \\
\hline Late gastrointestinal toxicity & & & 0.652 \\
\hline 0 & $37(86.0)$ & $37(88.1)$ & \\
\hline 2 & $2(4.7)$ & $3(7.1)$ & \\
\hline 3 & $4(9.3)$ & $2(4.8)$ & \\
\hline Hematological toxicity & & & 1 \\
\hline 0 & $20(46.5)$ & $19(45.2)$ & \\
\hline 1 & $15(34.9)$ & $15(35.7)$ & \\
\hline 2 & $7(16.3)$ & $7(16.7)$ & \\
\hline 3 & $1(2.3)$ & $1(2.4)$ & \\
\hline Acute hepatic toxicity & & & 1 \\
\hline 0 & $40(93.0)$ & $40(95.2)$ & \\
\hline 1 & $3(7.0)$ & $2(4.8)$ & \\
\hline \multicolumn{4}{|l|}{ Late hepatic toxicity } \\
\hline 0 & $29(67.4)$ & $33(78.6)$ & 0.011 \\
\hline 1 & $5(11.6)$ & $0(0.0)$ & \\
\hline 2 & $3(7.0)$ & $0(0.0)$ & \\
\hline 3 & $2(4.7)$ & 5 (11.9) & \\
\hline 4 & $3(7.0)$ & $1(2.4)$ & \\
\hline 5 & $1(2.3)$ & $3(7.1)$ & \\
\hline $0-2$ & $37(86.0)$ & $33(78.6)$ & 0.366 \\
\hline $3-5$ & $6(14.0)$ & $9(21.4)$ & \\
\hline
\end{tabular}

IG-IMRT, image-guided intensity-modulated radiotherapy; RTOG, Radiation Therapy Oncology Group.

failure, of which 29 patients [14 patients in non-IG-IMRT group $v s .15$ patients in image guided radiotherapy (IGRT) group] were caused by progression of intrahepatic tumors, while others were caused by RILD. The incidence of extrahepatic metastasis of non-IG-IMRT group $v s$. IGIMRT group was 7 (18.4\%) vs. 9 (22.5\%). There were three patients died of GI bleeding, one patient in IG-IMRT group $v s$. two in non-IG-IMRT group. Only one patient in this study died of pulmonary infarction.

\section{Discussion}

With the improvement of RT technologies, the radiation dose can be delivered much more precisely, without substantially increasing the dose to OARs. EBRT is a treatment option for all tumors irrespective of the location recommended by American 2017 NCCN guidelines of hepatobiliary cancers (20). In our previous studies, one study in 2005 collected HCC patients with LNM, patients in EBRT group achieved 9.4 months of OS compared with 3.3 month of non-EBRT group (12). Another study in 2013 analyzed 191 patients with LNM from HCC treated by EBRT provided a survival of 8.0 months (21). A report from Korea showed similar survival of 10.0 months (22). A series of studies shows EBRT is an effective treatment option for HCC patients with LNM and provides a noninvasive methodology.

In this study, we performed a retrospective analysis to evaluate responses and toxicities of IG-IMRT and non-IGIMRT for HCC patients with LNM. The median survival of patients in non-IG-IMRT group treated by 3D-CRT or IMRT techniques was 9.7 months, which is consistent with our former studies. Interestingly, patients in IG-IMRT group achieved a longer median survival of 15.3 months $(\mathrm{P}=0.098)$. A further analysis on 1 -year survival of the two groups showed $31.0 \%$ of improvement in IG-IMRT group with $\mathrm{P}=0.006$. IG-IMRT showed a short-term survival benefit at 1 year. Therefore, a large scale further research is definitely in need.

IG-IMRT is well known for its maximal accuracy and precision throughout the whole RT period by applying imaging guidance, which makes it possible to deliver higher radiation doses with less fractions. A dose-response relationship of RT in HCC patients has been reported from several studies (23-25). We summarized a list of clinical outcomes of EBRT for HCC patients with LNM in the past two decades, which is shown in Table 5. We can conclude that the median OS of patients in non-IG-IMRT group was within the range of 5.0-13.0 months and the range of median $\mathrm{BED}_{10}$ was from 39.0 to $75.0 \mathrm{~Gy}$. The median $\mathrm{BED}_{10}$ for IG-IMRT in this study was $67.2 \mathrm{~Gy}$ and for IMRT in Jang's study was 75 Gy (29). However, the median OS and 1, 2-year survival rate of patients in IMRT were still not as good as patients in IG-IMRT group compared with this study. In this study, there was no significant difference at 2-year survival, IG-IMRT vs. Non-IG-IMRT was $19.3 \%$ 
Table 5 Clinical outcomes of previous studies of EBRT for hepatocellular carcinoma patients with lymph node metastasis

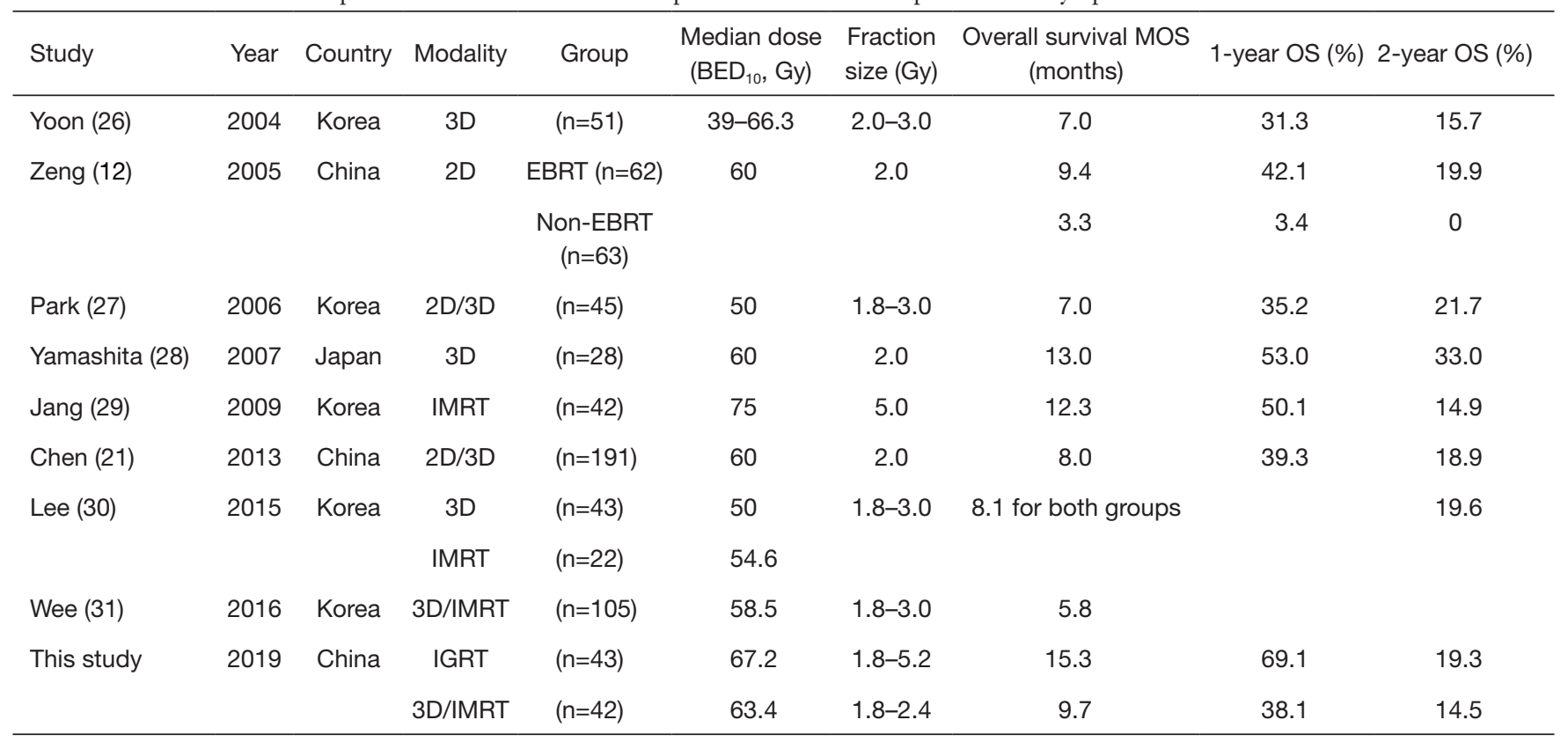

EBRT, external beam radiotherapy; IGRT, image guided radiotherapy; OS, overall survival.

vs. $14.5 \%, \mathrm{P}=0.066$. Seemingly, advanced techniques with improved radiation doses was not necessarily benefited long-term survival, and deserve further observation to draw definitive conclusions.

Tumor responses and the objective response rate (ORR) of the two groups analyzed in the present study showed no significance. Furthermore, we performed subgroup analyses to explore what kind of patients in the two groups benefited more from IG-IMRT based on ORR. We found that HCC patients with multiple LNMs achieved $100 \%$ ORR with IG-IMRT, while only $73.9 \%$ in the non-IG-IMRT group, $(\mathrm{P}=0.007)$. In this subgroup analysis, six patients $(26.1 \%)$ in the non-IG-IMRT group had SD, which indicated that IGIMRT had an advantage in patients with multiple targets. Although a less than $10 \%$ higher therapeutic dose was delivered to the IG-IMRT group [which made the group had a better 1-, and 2-year LCR compared to the non-IGIMRT group; 1 -year LCR was $74 \%$ vs. $52.3 \%$ and 2 -year LCR was $69 \%$ vs. $47 \%$, respectively $(\mathrm{P}=0.019)$ ], it provided a potential survival benefit. However, as a result of palliative radiation dose limitations by OARs, the dose was not high enough to improve long-term OS. Meanwhile, two patients $(5 \%)$ in the IG-IMRT group $v s$. four $(10.5 \%)$ in the nonIG-IMRT group died of lymph node-related complications induced by enlarged lymph nodes, such as obstructive jaundice, pyloric obstruction, intestinal paralysis, and inferior vena cava. The percentage of patients who died of lymph node-related complications in our study was consistent with $8 \%$ of patients reported by our previous studies.

As described above, patients in the IG-IMRT group received slightly higher radiation doses. However, the results of toxicity evaluations of acute and late GI toxicities, hematological toxicity, and acute hepatic toxicity were similar to those for the non-IG-IMRT group, which was delivered at relative low doses and no grade 4 or grade 5 toxicities were observed. Abdominal LNMs were usually surrounded by intestinal tract, which limited the dose escalation up to radical doses. To some extent, IG-IMRT relieved this limitation and improved short-term OS. This is consistent with Jiang's report about unresectable intrahepatic tumors in HCC patients (32) and Hou's report on HCC patients with portal vein and/or inferior vena cava tumor thrombi (33). Nevertheless, the reason why less than $10 \%$ dose escalation of BED can significantly improve short-term OS remains unclear, and further study is necessary to achieve definitive conclusions.

One patient $(2.3 \%)$ in the IG-IMRT group and three patients $(7.1 \%)$ in the non-IG-IMRT group died of liver failure is the main cause of death for HCC patients with LNM in this study. Liver failure caused by hepatotoxicity is definitely different from tumor progression which can be 
differentiated by RILD mentioned above. It showed similar dose distribution between the two groups except for the percentage of whole liver covered by at least 5 Gy (V5) (33). Furthermore, we compared the treatment plan of one patient treated by IG-IMRT or non-IG-IMRT from the respect of a RT oncologist. The dose distribution from V5V30 and mean dose of normal liver in IG-IMRT treatment plan are less than CRT. The protection of OARs including stomach, intestine and kidneys in IG-IMRT treatment plan is better than CRT. Furthermore, the conformal index and homogeneity index are both better in IG-IMRT treatment plan than CRT. With the dose permissible by OARs and normal liver, the IG-IMRT group yielded a successful dose escalation. In addition, patients in the IG-IMRT group had at least a 1-week shorter course of treatment than did patients in the non-IG-IMRT group.

In this study, the following limitations should be considered. First, the survival analysis is limited by retrospective nature and small number of patients. Second, treatment selection depends on patients' economics greatly. IG-IMRT has not included in medical insurance in China during the period of our study. Third, it is very uncommon for patients with LNM from HCC, it is difficult to reach large scale of patients. Therefore, a further multi-center and random clinical trial on the effect of IG-IMRT and non-IG-IMRT is definitely necessary to get a confident conclusion.

\section{Conclusions}

The therapeutic dose delivered by IG-IMRT is slightly higher than non-IG-IMRT which was more effective and showed superior short-term survival and local control in HCC patients with LNM.

\section{Acknowledgments}

Funding: This work was supported by a Scientific Research Project of Shanghai Science and Technology Commission (Grant No. 17411962600).

\section{Footnote}

Conflicts of Interest: The authors have no conflicts of interest to declare.

Ethical Statement: The authors are accountable for all aspects of the work in ensuring that questions related to the accuracy or integrity of any part of the work are appropriately investigated and resolved. This study was approved by the Ethics Committee of Fudan University Zhongshan Hospital (2011-235).

\section{References}

1. Sun HC, Zhuang PY, Qin LX, et al. Incidence and prognostic values of lymph node metastasis in operable hepatocellular carcinoma and evaluation of routine complete lymphadenectomy. J Surg Oncol 2007;96:37-45.

2. Ercolani G, Grazi GL, Ravaioli M, et al. The role of lymphadenectomy for liver tumors: further considerations on the appropriateness of treatment strategy. Ann Surg 2004;239:202-9.

3. Primary liver cancer in Japan. Sixth report. The Liver Cancer Study Group of Japan. Cancer1987;60:1400-11.

4. Primary liver cancer in Japan. Clinicopathologic features and results of surgical treatment. Ann Surg 1990;211:277-87.

5. Yuki K, Hirohashi S, Sakamoto M, et al. Growth and spread of hepatocellular carcinoma. A review of 240 consecutive autopsy cases. Cancer 1990;66:2174-9.

6. Watanabe J, Nakashima O, Kojiro M. Clinicopathologic study on lymph node metastasis of hepatocellular carcinoma: a retrospective study of 660 consecutive autopsy cases. Jpn J Clin Oncol 1994;24:37-41.

7. Poon RT, Fan ST, Tsang FH, et al. Locoregional therapies for hepatocellular carcinoma: a critical review from the surgeon's perspective. Ann Surg 2002;235:466-86.

8. European Association for the Study of the Liver, European Organisation for Research and Treatment of Cancer. EASL-EORTC clinical practice guidelines: management of hepatocellular carcinoma. J Hepatol 2012;56:908-43.

9. Ministry of Public Health of the People's Republic of China. Guideline for diagnosis and treatments of hepatocellular carcinoma. Chin Clin Oncol 2011;16:929-46.

10. Korean Liver Cancer Study Group and National Cancer Center, Korea. Practice guidelines for management of hepatocellular carcinoma 2009 (in Korean). Korean J Hepatol 2009;15:391-423.

11. Zeng ZC, Tang ZY, Yang BH, et al. Radiation therapy for the locoregional lymph node metastases from hepatocellular carcinoma, phase I clinical trial. Hepatogastroenterology 2004;51:201-7.

12. Zeng ZC, Tang ZY, Fan J, et al. Consideration of role of radiotherapy for lymph node metastases in patients with HCC: retrospective analysis for prognostic factors from 125 
patients. Int J Radiat Oncol Biol Phys 2005;63:1067-76.

13. Zhou J, Sun HC, Wang Z, et al. Guidelines for Diagnosis and Treatment of Primary Liver Cancer in China (2017 Edition). Liver Cancer 2018;7:235-60.

14. Hu Y, Zhou YK, Chen YX, et al. Does liver resection/ transplantation affect respiratory induced liver motion in patients with hepatocellular carcinoma? J Appl Clin Med Phys 2017;18:185-92.

15. Wang MH, Ji Y, Zeng ZC, et al. Impact factors for microinvasion in patients with hepatocellular carcinoma: possible application to the definition of clinical tumor volume. Int J Radiat Oncol Biol Phys 2010;76:467-76.

16. Eisenhauer EA, Therasse P, Bogaerts J, et al. New response evaluation criteria in solid tumours: revised RECIST guideline (version 1.1). Eur J Cancer 2009;45:228-47.

17. Cox JD, Stetz J, Pajak TF. Toxicity criteria of the Radiation Therapy Oncology Group (RTOG) and the European Organization for Research and Treatment of Cancer (EORTC). Int J Radiat Oncol Biol Phys 1995;31:1341-6.

18. Pan CC, Kavanagh BD, Dawson LA, et al. Radiationassociated liver injury. Int J Radiat Oncol Biol Phys 2010;76:S94-100.

19. Olsen CC, Welsh J, Kavanagh BD, et al. Microscopic and macroscopic tumor and parenchymal effects of liver stereotactic body radiotherapy. Int J Radiat Oncol Biol Phys 2009;73:1414-24.

20. Benson AB 3rd, D'Angelica MI, Abbott DE, et al. NCCN Guidelines Insights: Hepatobiliary Cancers, Version 1.2017. J Natl Compr Canc Netw 2017;15:563-73.

21. Chen YX, Zeng ZC, Fan J, et al. Defining prognostic factors of survival after external beam radiotherapy treatment of hepatocellular carcinoma with lymph node metastases. Clin Transl Oncol 2013;15:732-40.

22. Kim K, Chie EK, Kim W, et al. Absence of symptom and intact liver function are positive prognosticators for patients undergoing radiotherapy for lymph node metastasis from hepatocellular carcinoma. Int J Radiat Oncol Biol Phys 2010;78:729-34.

23. Park HC, Seong J, Han KH, et al. Dose-response relationship in local radiotherapy for hepatocellular

Cite this article as: Zhang $\mathrm{H}$, Chen $\mathrm{Y}$, Hu Y, Yang P, Wang B, Zhang J, Sun J, Zeng Z. Image-guided intensitymodulated radiotherapy improves short-term survival for abdominal lymph node metastases from hepatocellular carcinoma. Ann Palliat Med 2019;8(5):717-727. doi: 10.21037/ apm.2019.11.17 carcinoma. Int J Radiat Oncol Biol Phys 2002;54:150-5.

24. Park W, Lim DH, Paik SW, et al. Local radiotherapy for patients with unresectable hepatocellular carcinoma. Int J Radiat Oncol Biol Phys 2005;61:1143-50.

25. Kim DY, Park W, Lim DH, et al. Three-dimensional conformal radiotherapy for portal vein thrombosis of hepatocellular carcinoma. Cancer 2005;103:2419-26.

26. Yoon SM, Kim JH, Choi EK, et al. Radioresponse of hepatocellular carcinoma-treatment of lymph node metastasis. Cancer Res Treat 2004;36:79-84.

27. Park YJ, Lim DH, Paik SW, et al. Radiation therapy for abdominal lymph node metastasis from hepatocellular carcinoma. J Gastroenterol 2006;41:1099-106.

28. Yamashita H, Nakagawa K, Shiraishi K, et al. Radiotherapy for lymph node metastases in patients with hepatocellular carcinoma: retrospective study. J Gastroenterol Hepatol 2007;22:523-7.

29. Jang JW, Kay CS, You CR, et al. Simultaneous multitarget irradiation using helical tomotherapy for advanced hepatocellular carcinoma with multiple extrahepatic metastases. Int J Radiat Oncol Biol Phys 2009;74:412-8.

30. Lee DY, Park JW, Kim TH, et al. Prognostic indicators for radiotherapy of abdominal lymph node metastases from hepatocellular carcinoma. Strahlenther Onkol 2015;191:835-44.

31. Wee CW, Kim K, Chie EK, et al. Prognostic stratification and nomogram for survival prediction in hepatocellular carcinoma patients treated with radiotherapy for lymph node metastasis. Br J Radiol 2016;89:20160383.

32. Jiang T, Zeng ZC, Yang P, et al. Exploration of Superior Modality: Safety and Efficacy of Hypofractioned Image-

Guided Intensity Modulated Radiation Therapy in Patients with Unresectable but Confined Intrahepatic Hepatocellular Carcinoma. Can J Gastroenterol Hepatol 2017;2017:6267981.

33. Hou JZ, Zeng ZC, Wang BL, et al. High dose radiotherapy with image-guided hypo-IMRT for hepatocellular carcinoma with portal vein and/or inferior vena cava tumor thrombi is more feasible and efficacious than conventional 3D-CRT. Jpn J Clin Oncol 2016;46:357-62. 